\title{
GENETICS OF THE DEMENTIAS
}

J M Schott, N C Fox, M N Rossor

J Neurol Neurosurg Psychiatry 2002;73(Suppl II):ii27-ii3 1

W hile the majority of dementias are sporadic, many diseases causing dementia can also occur on a familial basis. Some of these show an autosomal or sex linked inheritance. In others, certain genes are not directly causative, but are major risk factors. Some dementias may be definitively diagnosed in vivo or prenatally using genetic testing. The discovery of causative genes has also led to the development of cellular and animal models of many of these diseases. Table 1 lists the hereditary dementias discussed in this article.

\section{ALZHEIMER'S DISEASE}

\section{Familial Alzheimer's disease}

Alzheimer's disease (AD), the most common cause of dementia, is characterised by the accumulation of neurofibrillary tangles and neuritic amyloid plaques. These histopathological changes lead to progressive cerebral atrophy and cognitive decline. While increasing age is the single greatest risk factor for the development of $\mathrm{AD}, \mathrm{AD}$ in a first degree relative confers a doubling of the relative risk for an individual. In less than $5 \%$ of cases $\mathrm{AD}$ is inherited in an autosomal dominant manner with almost complete penetrance. Such autosomal dominant familial AD (FAD) is associated with mutations in three genes: $\beta$-amyloid precursor protein (APP) gene, presenilin 1, and presenilin 2 . (An up to date list of all known mutations in these three genes is found at http://molgenwww.uia.ac.be/Admutations.) In some patients with FAD a definite mutation may not be found.

\section{$\beta$-Amyloid precursor protein}

The APP gene, located on the long arm of chromosome 21 , encodes an alternatively spliced transcript that, in its longest isoform, expresses a single transmembrane polypeptide of 770 amino acids. APP is processed by two major pathways. The first, controlled by $\alpha$-secretase, produces a non-amyloidogenic protein product. The second, combining the sequential action of $\beta$ - and $\gamma$-secretases, generates $A \beta$ peptides of 40-43 amino acids; the accumulation of $A \beta-42$, a highly amyloidogenic protein and the most abundant species within neuritic plaques, is central to the amyloid cascade hypothesis of AD.

The first mutation in the APP gene causing familial AD was described in 1991, and subsequently several causative mutations in the APP gene have been identified, all producing an increase in A $\beta-42$ by different mechanisms; nonetheless, APP mutations account for less than $5 \%$ of cases of early onset FAD. All the known pathogenic mutations are centred around the $\alpha-, \beta-$, or $\gamma$-secretase sites, and either increase the production of the longer $A \beta-42$, or both the long and short forms of $A \beta$. The increased production of APP and A $\beta-42$ in Down's syndrome is caused by the extra copy of chromosome 21 , and is also associated with early onset AD. One specific mutation in the $\beta$-APP gene (glycine for glutamate at codon 693) is associated with hereditary cerebral haemorrhage with amyloidosis of the Dutch type (HCHWA-D), in which patients present with cerebral haemorrhage, in the absence of neuritic pathology. Another mutation, the "Arctic mutation" described in a Swedish family, appears to cause AD by increasing $\mathrm{A} \beta$ protofibril formation, leading to an accelerated build-up of insoluble protein.

Within a given family with an APP mutation, the age at onset is often remarkably consistent, and is generally between $45-60$ years. While patients with certain mutations may have characteristic clinical features (such as myoclonus or seizures), there are no consistent clinical features that reliably distinguish patients with APP mutations from patients with either sporadic AD or FAD caused by other mutations. It is likely that Apo-E genotype may influence the age at onset (see below). Genetic screening for APP mutation generally assesses exons 16 and 17, the sites of the common mutations.

Correspondence to: Professor MN Rossor, Dementia Research Group, Box 16, National Hospital for Neurology and Neurosurgery, Institute of Neurology, Queen Square, London WCIN 3BG;

m.rossor@dementia.ion.ucl.ac.uk

\section{Presenilins}

Despite the fact that mutations in presenilin-1 (PS-1) are far more common than those in APP as a cause of FAD, the first PS-1 mutation causing familial AD was not described until 1995. Approximately $50 \%$ of early onset FAD cases are associated with mutations in PS-1, and over 100 mutations 


\section{Abbreviations}

$\beta$-APP: $\beta$-amyloid precursor protein

AD: Alzheimer's disease

ApoE: apolipoprotein $\mathrm{E}$

CADASIL: cerebral autosomal dominant arteriopathy with subcortical infarcts and leucoencephalopathy

CJD: familial Creutzfeldt-Jakob disease

FAD: familial Alzheimer's disease

FENIB: familial encephalopathy with neuroserpin inclusion bodies

FFI: fatal familial insomnia

FTDP-1: frontotemporal dementia with parkinsonism linked to chromosome 17

FTLD: frontotemporal lobar degeneration

GSS: Gerstmann-Sträussler-Scheinker

HCHWA-D: hereditary cerebral haemorrhage with amyloidosis of the Dutch type

HD: Huntington's disease

$\operatorname{PrP}^{\mathrm{C}}$ : normal prion protein

$\operatorname{PrP}^{\mathrm{Sc}}$ : abnormal prion protein (scrapie agent)

PS-1: presenilin 1

PS-2: presenilin 2

have been described. Evidence from cell culture and postmortem findings supports the hypothesis that PS I mutations lead to overproduction of A $\beta-42$. PS- 2 mutations are rarer $(<1 \%$ of all cases of FAD). The presenilin genes, located on chromosome 14 (PS-1), and chromosome 1 (PS-2), show a high degree of homology. While the function of their protein products is uncertain, there is evidence to implicate the presenilins in the $\gamma$-secretase proteolysis of $\beta$-APP; indeed it has been postulated that presenilin 1 actually is the $\gamma$-secretase.

The clinical spectrum of presenilin mutations is wide, with the reported age at onset varying from $20-60$ years. Although early age at onset and faster progression are the rule, there may be dramatic differences in clinical presentation within the same kindred; unlike APP mutations, ApoE genotype may not influence delay or non-penetrance in PS-1 mutations. Some families have specific phenotypes, such as a spastic paraparesis or prominent myoclonus, although these features do not seem to be specific for a given mutation; mutations in certain sites within the PS-1 gene may result in increased angiopathy. Families with PS-2 mutations may have an older age of onset than those with PS-1, with a reported age range of between 50-60 years. Genetic screening for PS mutations generally assesses exons 2 to 12 (PS-1) and 4, 5, and 7 (PS-2), the sites of the common mutations.

\section{Sporadic Alzheimer's disease}

The epidemiology of the much more common, late onset "sporadic"' $\mathrm{AD}$ is complex. No single gene is causative, and it is likely that multiple combinations of risk factors, both genetic and environmental, could result in AD. There is, however, evidence for the involvement of certain genes in the development of sporadic AD. The gene coding for apoliopoprotein E (ApoE) exists in three allelic forms: $\epsilon 2, \epsilon 3$, and $\epsilon 4$. Caucasians carrying the $\epsilon 4$ allele are three (heterozygotes) to eight (homozygotes) times more likely to develop AD than individuals without an $\epsilon 4$ allele. Nonetheless, between $40-70 \%$ of patients with late onset $\mathrm{AD}$ do not carry the $\epsilon 4$ allele, and significant proportions of the normal population carry the $\epsilon 4$ allele without any cognitive impairment. There is, therefore, no clinical role for ApoE genotyping outside of a research setting. Similarly, while linkage research has implicated areas on chromosomes 6, 9, 10,

\begin{tabular}{lll} 
Table 1 Hereditary dementias & & \\
\hline Disease & Gene & Chromosome \\
\hline Familial Alzheimer's disease & $\beta$-APP & 21 \\
& PS-1 & 14 \\
& PS-2 & 1 \\
Sporadic Alzheimer's disease & ApoE & 19 \\
Hereditary cerebral haemorrhage with & $\beta$-APP & 21 \\
amyloidosis of the Dutch type & & \\
Familial Creutzfeldt Jakob disease & Prion & 20 \\
Familial fatal insomnia & Prion & 20 \\
Gerstmann-Sträussler-Scheinker & Prion & 20 \\
FTDP-17 & Tau & 17 \\
Frontotemporal dementia (Jutland) & $?$ & 3 \\
Frontotemporal dementia & $?$ & 9 \\
Huntington's disease & IT-15 & 4 \\
CADASIL & Notch 3 & 19 \\
Familial British dementia & BRI2 & 13 \\
Familial Danish dementia & BRI2 & 13 \\
FENIB & PI12 & 3 \\
\hline
\end{tabular}

and 12 as loci harbouring potential risk genes for $\mathrm{AD}$, such work has yet to find a clinical role.

\section{PRION DISEASES}

Prion diseases may be sporadic, inherited or transmissible. The core feature of all prion diseases is the post-translational conversion of a normal human protein, the prion protein $\left(\operatorname{PrP}^{\mathrm{C}}\right)$, to an abnormal form, designated $\operatorname{PrP}^{\mathrm{sc}}$. The function of normal human prion protein is unknown, but it is coded for by a single exon on the long arm of chromosome 20 (PRNP gene). The histological features of prion diseases are of cortical and subcortical spongiform change, neuronal loss, and deposition of $\operatorname{PrP}^{\mathrm{SC}}$.

Prion diseases are rare (with a worldwide incidence of approximately 1 case per million), and inherited forms of prion disease are rarer still, accounting for around 15\% of all human prion diseases. Such diseases are usually associated with point mutations or insertions in the prion gene. Three phenotypes have traditionally been recognised: familial Creutzfeldt-Jakob disease (CJD), Gerstmann-SträusslerScheinker (GSS) syndrome, and fatal familial insomnia (FFI). All show autosomal dominant inheritance, are experimentally transmissible, and "typical"' features of sporadic CJD, such as myoclonus and periodic EEG activity, may be absent. The increasing availability of molecular diagnostic techniques, and the large overlap in phenotype both between the conditions, and between family members with the same mutation, has increasingly led to classifications based on mutation alone. Importantly, at least two mutations in the prion gene (at codons 145 and 183) may cause a disease that clinically mimics $\mathrm{AD}$, and an insertion at base pair 144 may present with a very variable phenotype. As described below, the clinical phenotype may also be dramatically influenced by polymorphisms at codon 129.

\section{Familial Creutzfeldt-Jakob disease}

The most common mutation associated with familial CJD is at codon 200 of the prion gene. Apart from a slightly earlier 
average age at onset (55 years), the disease is clinically indistinguishable from the sporadic form of CJD. Nearby mutations at codons 208 and 210 in Italian families produce a similar phenotype; all three mutations exhibit incomplete penetrance. The second most common mutation, at codon 178, produces a disease with an earlier onset (fifth decade) and longer duration ( $1-2$ years). Other point mutations have been described in only one or two families.

\section{Gerstmann-Sträussler-Scheinker syndrome}

The majority of affected families with GSS have a point mutation at codon 102. Onset is in the third or fourth decade, and the disease runs a course over several years. Patients initially present with cerebellar dysfunction, and subsequently develop dementia. Extrapyramidal features, gaze palsies, deafness, pseudobulbar palsy (codon 117 mutation), and cortical blindness may be present in some families; examination findings may include depressed reflexes and extensor plantars.

\section{Fatal familial insomnia}

Progressive insomnia associated with dysautonomia, motor signs, and memory impairment are the hallmarks of FFI. The age at onset may vary from 20-70 years, with a variable phenotype at least partly associated with polymorphisms at codon 129. The memory impairment is said to be characteristic, with severe impairments of attention and memory, in the face of good preservation of general intellectual functioning. The disease is associated with the same mutation at codon 178 that produces familial CJD. Whether this mutation leads to FFI or CJD is influenced by the polymorphic variation at codon 129: when it encodes valine on the mutant 178 allele, the result is CJD; when it encodes methionine, the result is FFI. To compound difficulties of genotype/phenotype correlation, an identical syndrome has been reported in a patient with a mutation at codon 200, and in patients with sporadic prion disease.

Hereditary prion diseases exemplify many of the problems associated with inconsistent genotype-phenotype correlations. A screen for mutations in the prion gene is therefore indicated if the clinical picture is suspicious, and particularly if a family history is obtained. Such testing generally involves sequencing the whole open reading frame of the prion gene (approximately 100 base pairs)

\section{Variant and sporadic CJD}

While variant CJD has been linked to transmission of the agent of bovine spongiform encephalopathy, all cases tested to date have been homozygous for methionine at codon 129. The heterogeneity in clinical presentation of sporadic CJD is likely to be determined both by the physico-chemical properties of $\mathrm{PrP}^{\mathrm{SC}}$ and codon 129 genotype; however, such polymorphism testing currently remains a research tool.

\section{FRONTOTEMPORAL LOBAR DEGENERATION}

Frontotemporal lobar degeneration (FTLD) is a heterogeneous condition, characterised by behavioural and personality changes, progressive dementia, variable movement disorders, and language impairment. Up to $50 \%$ of patients have a positive family history.

\section{Frontotemporal dementia with parkinsonism linked to chromosome 17}

At a consensus meeting in 1996 many previously described families with diverse autosomal dominant conditions linked to chromosome 17 were classified together. Subsequently,
UK associations and societies providing information for patients and carers

Alzheimer's Society

Gordon House, 10 Greencoat Place, London SW1P 1 PH. Tel: Helpline 0845300 0336; fax: 02073060808 ; email: info@alzheimers.org.uk; www.alzheimers.org.uk

Creutzfeldt-Jakob Disease Support Network

National CJD Co-ordinator, Birchwood, Heath Top, Ashley Heath, Market Drayton, Salop, TF9 4QR. Tel: CJD helpline: 01630 673973; fax: 01630673993

\section{Huntington's Disease Association}

108 Battersea High Street, London SW 11 3HP. Tel: 0207223

7000; fax: 0207223 9489; email: info@hda.org.uk; www.hda.org.uk

Scottish Huntington's Association

Thistle House, 61 Main Road, Elderslie, Johnstone, PA5 9BA.

Tel: 01505 322245; fax: 01505382980 ;

email: sha-admin@hdscotland.org; www.hdscotland.org

Pick's Disease Support Group

3 Fairfield Park, Lyme Regis, Dorset DT7 3DS.

Email: Penelope@pdsg.org.uk; www.pdsg.org.uk

causative genetic mutations have been demonstrated in the microtubule associated protein tau (MAPtau) gene on chromosome 17q21. Frontotemporal dementia with parkinsonism linked to chromosome 17 (FTDP-17) is strikingly heterogeneous; common features include a predominantly frontotemporal distribution of pathology, pronounced behavioural change, and extrapyramidal dysfunction; some affected families have psychotic symptoms, and some amyotrophy. To date over 20 pathological mutations have been reported; most mutations increase the number of 4-repeat tau isoforms; others alter tau-microtubule binding. The large heterogeneity of the condition is likely to be the consequence of both the effects of the different mutations, and of other, as yet unknown factors; indeed the same mutation in the same family can result in remarkably different clinical phenotypes and ages at onset.

\section{Other genetic causes of FTLD}

Other families with autosomal dominant FTLD have been linked to chromosome 9, and in one large Danish kindred from Jutland, familial autosomal dominant FTD has been linked to chromosome 3. Familial FTLD with ubiquitinpositive, tau-negative inclusions on histology has been described. In these families, linkage to chromosome 17q21-22 has been demonstrated in the absence of mutations in the MAPtau gene.

To date, while mutation screening may be available for families with FTDP-17, screening for tau mutations is generally restricted to research settings.

\section{OTHER GENETIC CAUSES OF DEMENTIA}

Many genetic diseases may present with dementia as part of the clinical phenotype. Some of the diseases, where dementia is a prominent if not sole manifestation, are discussed below. Some other hereditary diseases where dementia may be a feature are listed in table 2, and are discussed in more detail elsewhere in this supplement.

\section{Huntington's disease}

Huntington's disease (HD) is an autosomal dominant disease, with onset most frequently in middle age. Typical symptoms 
Table 2 Some hereditary diseases that may present with dementia in adulthood

\begin{tabular}{ll}
\hline & Inheritance \\
\hline Adrenoleukodystrophy & X linked \\
Dentatorubropallidoluysian atrophy & Autosomal dominant \\
Mitochondrial disorders (MERFF, MELAS, Leighs) & Maternal \\
Niemann Pick type C & Autosomal recessive \\
Parkinson's disease & Autosomal dominant \\
Spinocerebellar ataxia & Autosomal Dominant \\
Wilson's disease & Autosomal Recessive \\
\hline
\end{tabular}

Most of these diseases are discussed elsewhere in this supplement.

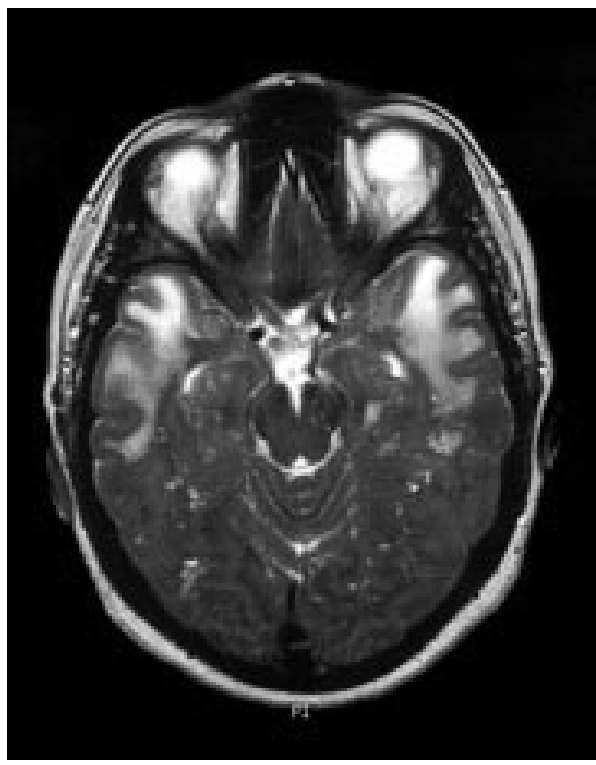

Figure 1 Magnetic resonance image of a patient with cerebral autosomal dominant arteriopathy with subcortical infarcts and leucoencephalopathy (CADASIL).

include movement disorders (classically chorea), a predominantly subcortical cognitive impairment, and psychiatric disturbances. The genetic abnormality consists of an expansion of the trinucleotide repeat CAG in the gene IT- 15 on chromosome 4. Normal repeat sizes are 10-35; repeat lengths $>39$ will result in manifest HD in a person who lives a normal life span. Age at onset is inversely proportional to length of CAG repeat, and the disease shows anticipation. A direct HD gene test is available and is highly sensitive and specific. The guidelines for presymptomatic testing in HD provide useful guidance for the testing of other autosomal dominant conditions, including FAD.

\section{Cerebral autosomal dominant arteriopathy with subcortical infarcts and leucoencephalopathy}

Cerebral autosomal dominant arteriopathy with subcortical infarcts and leucoencephalopathy (CADASIL) is a rare familial cause of vascular dementia and stroke in young adults, with mean age at onset of 45 years. The clinical picture may be associated with migraine with aura and frontal cognitive disturbances. Magnetic resonance imaging (MRI) reveals characteristic anterior temporal white matter change (fig 1), with diffuse white matter lesions in the cerebral hemispheres, basal ganglia, and pons. The causative mutation is in the Notch 3 gene on chromosome 19p13.1. The disease can be diagnosed by detecting granular osmiophilic material detected

\section{Useful websites for health care professionals}

- Gene testing laboratories in the UK: http://www.cmgs.org

- Directory of UK genetics centres: http://www.bshg.org.uk/ Directory/UKdirectory.htm

- AD mutation list: http://molgen-www.uia.ac.be/Admutations/

- $A D$ research including mutation information: http:// www.alzforum.org/members/index.html

- Directory of genetic disorders: http://www.geneclinics.org/

by electron microscopy in proximity to vascular smooth muscle cells in skin, muscle, peripheral nerve, and brain. Genetic testing is also possible; exons 3 and 4 of the Notch 3 gene harbour $70 \%$ of the mutations, and are therefore the first areas to be assessed. It is possible to screen further exons if clinically indicated.

\section{Familial British and Danish dementias}

Familial British dementia with amyloid angiopathy is a rare autosomal dominant condition characterised by dementia, progressive spastic tetraparesis, and cerebellar ataxia with onset in the sixth decade. A point mutation in the BRI gene on chromosome 13 has been shown to be the genetic cause of the disease. A duplication in this gene causes the related familial Danish dementia characterised by cataracts, deafness, progressive ataxia, and dementia.

\section{Familial encephalopathy with neuroserpin inclusion bodies}

A novel autosomal dominant disease caused by a point mutation in the gene coding for neuroserpin (on chromosome 3) has been recently described. Familial encephalopathy with neuroserpin inclusion bodies (FENIB) is associated with a progressive dementia, with relative sparing of recall memory, and histologically by the accumulation of cytoplasmic inclusions within the deep cortical layers, substantia nigra, and subcortical nuclei.

\section{CLINICAL ASPECTS}

The possibility of a familial form of dementia should be considered in all patients presenting with dementia at a young age. As specific features suggestive of a familial dementia may not be present, an accurate and complete family history is an essential part of the assessment of any patient with a dementia. It may be difficult to extract a reliable history from a cognitively impaired patient, and, providing consent is given, it may be necessary to request further information from a relative or carer. Particular care should be taken not to overlook the early death of a family member who might have subsequently developed dementia.

Genetic testing may be appropriate in symptomatic patients in whom there is a family history of autosomal dominant disease, or in patients with an appropriate clinical phenotype. Screening for mutations may have implications for insurance and employment, as well as for other family members, and there may be particular issues in a cognitively impaired patient group. Consequently, such tests should be performed after appropriate counselling in conjunction with clinical geneticists or in specialist centres; written consent should also be obtained.

Mutation screening in affected individuals with a clear family history of dementia can be guided by the clinical phenotype; clearly if there is a known mutation in the family, then the presence of this mutation can be directly assessed. All 
requests for genetic tests or counselling should be accompanied by as detailed a family history as possible. If the family history is less clear, there should be a good clinical reason for performing the test (such as the presence of MRI changes consistent with a diagnosis of CADASIL), otherwise the likelihood of gaining useful clinical information is much reduced, particularly in diseases where mutations may be present in multiple exons which cannot all be screened routinely. Likewise, the result of mutation testing needs to be read in conjunction with the particular test requested. Thus a negative test for HD virtually excludes the condition, while the absence of a mutation in the $\beta$-APP, presenilin 1 , or presenilin 2 genes by no means excludes the diagnosis of FAD. The International Huntington's Disease Society and the World Federation of Neurology have issued guidelines for presymptomatic diagnosis for HD, and these guidelines have been adopted for testing in FAD.

The postmortem diagnosis of the cause of a familial dementia may provide critically important information for future counselling for the family, and issues surrounding the postmortem examination should be discussed with patients and, where appropriate, family members. In patients with families with known mutations, it may now be possible to undertake prenatal diagnosis. There are ethical considerations in undertaking such testing for conditions that have no curative treatments, and do not manifest themselves until adult life; such testing should only be considered in specialist centres where genetic counselling is available.

\section{ACKNOWLEDGEMENTS}

The authors are grateful to Professor John Hardy for his helpful comments.

\section{Authors' affiliations}

J M Schott, N C Fox, *M N Rossor, Dementia Research Group, National Hospital for Neurology and Neurosurgery, Institute of

Neurology, Queen Square, London, UK

*Also Division of Neuroscience and Psychological Medicine, Faculty of Medicine, Imperial College of Science, Engineering and Medicine, London, UK

\section{KEY REFERENCES}

1 Hodges J (ed). Early-onset dementia. A multidisciplinary approach. Oxford: Oxford University Press, 2001.

An overview of the hereditary dementias.

2 Tandon A, Rogaeva E, Mullan M, et al. Molecular genetics of Alzheimer's disease: the role of beta-amyloid and the presenilins. Curr Opin Neurol 2000;13:377-84.

- Familial AD.

3 Myers AJ, Goate AM. The genetics of late-onset Alzheimer's disease. Curr Opin Neurol 2001;14:433-40.

Sporadic AD.

4 Dartigues JF, Letenneur L. Genetic epidemiology of Alzheimer's disease. Curr Opin Neurol 2000;13:385-9.

- Also sporadic AD.

5 Collinge J. Prion diseases of humans and animals: their causes and molecular basis. Annu Rev Neurosci 2001 ;24:519-50.

Prion diseases.

6 Lee VM, Goedert M, Trojanowski JQ. Neurodegenerative tauopathies. Annu Rev Neurosci 2001;24:1121-59.

Tau pathology: FTDP-17.

7 Davies S, Ramsden DB. Huntington's disease. J Mol Pathol 2001;54:409-13.

Huntington's disease.

8 Kalaria RN. Advances in molecular genetics and pathology of cerebrovascular disorders. Trends Neurosci 2001;24:392-400

- CADASIL.

9 Hardy J. Pathways to primary neurodegenerative disease. Ann N Y Acad Sci 2000;924:29-34.

- From genetics to pathogenesis.

10 International Huntington Association, and the World Federation of Neurology Research Group on Huntington's Chorea. Guidelines for the molecular genetics predictive test in Huntington's disease. J Med Genet 1994;31:555-9.

- Pre-symptomatic testing.

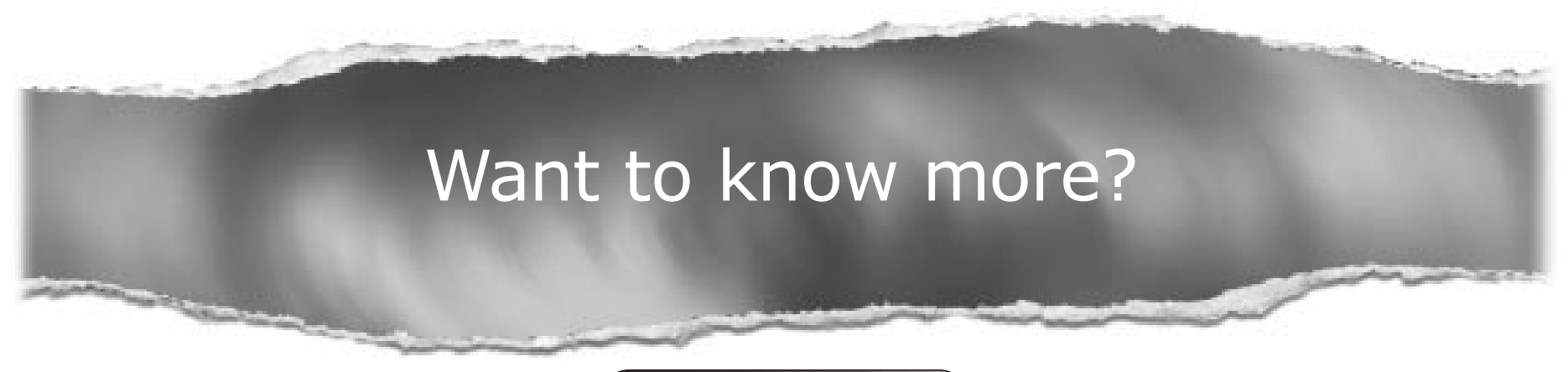

Data supplements

Limited space in printed journals means that interesting data and other material are often edited out of articles; however, limitless cyberspace means that we can include this information online.

Look out for additional tables, references, illustrations.

www.jnnp.com 


\section{NEUROMUSCULAR DISEASE}

Geraint Fuller, Ian Bone

J Neurol Neurosurg Psychiatry 2003;74(Suppl II):ii 1-ii2

$T$ his supplement tackles the lower levels of the nervous system, the peripheral nerves, the neuromuscular junction, and muscle diseases. Motor neurone disease/amyotrophic lateral sclerosis, a disorder of both upper and lower motor neurones, will be considered in a later supplement.

There are many diseases of nerve and muscle; a few are common, many are rare, a situation that often leaves neurologists with uncertainty as to how extensively to investigate their patients. Some of these conditions are treatable, though in none is this simple. Here we have chosen to emphasise the practical approach to the assessment and management of patients who present with neuromuscular symptoms.

Hugh Willison and John Winer outline their clinical approach to evaluating and investigating peripheral neuropathies, the evaluation of genetically determined neuromuscular disease having been discussed by Mike Hanna and Mary Reilly in the recent issue of Neurology in Practice on neurogenetics (see below). When a diagnosis of an inflammatory neuropathy is made there are a number of therapeutic options; Robert Hadden and Richard Hughes review what is available and the evidence for how and when it is used. Many patients with neuropathies are found to have diabetes. This is usually the first step in their evaluation causation and Gareth Llewellyn explores the types of diabetic neuropathies and their management. Focal peripheral neuropathies are very common, on the orthopaedic border of neurology, and Geraint Fuller outlines a practical approach.

While muscular symptoms are commonly complained of, only infrequently do they reflect significant muscular disease; Richard Petty discusses the symptomatic approach to evaluating patients with possible muscle diseases. David Hilton-Jones provides insights into the rare but difficult management of inflammatory muscle diseases. Bridging "the gap", Marguerite Hill discusses disorders of the neuromuscular junction, their diagnosis and management.

Much of the management of neuromuscular disease has evolved without being subjected to evaluation in clinical trials. Kate Jewitt and Richard Hughes provide a Cochrane view of how this is changing.

The route map below, as always, tries to highlight some useful references.

\section{KEY REFERENCES}

\section{Genetic neuromuscular disease}

- Genetic neuromuscular disease. Reilly MM, Hanna MG. J Neurol Neurosurg Psychiatry 2002;73(suppl II):iil2-21.

Clinical and laboratory approach to genetic neuromuscular disease.

\section{Neuropathies}

Guillain-Barré syndrome (GBS)

- Guillain-Barré syndrome. Hahn AF. Lancet 1998;352:635-41.

- Pathogenesis of Guillain-Barré syndrome. Hughes RAC, Hadden RDM, Gregson NA, Smith KJ. J Neuroimmunol 1999;100:74-97.

A detailed review on the cellular and antibody mediated mechanisms of pathogenesis of GuillainBarré syndrome.

Correspondence to: Dr GN Fuller, Department of Neurology, Gloucester Royal Hospital, Great Western Road, Gloucester GL1 3NN, UK: geraint@Fullerg.demon.co.uk
Chronic inflammatory demyelinating polyradiculoneuropathy (CIDP)

- Chronic inflammatory demyelinating polyradiculoneuropathy. Pollard JD. Curr Opin Neurol 2002;15:279-83.

Multifocal motor neuropathy (MMN)

- Multifocal motor neuropathy. Nobile-Orazio E. J Neuroimmunol 2001;115:4-18. 
Paraproteinaemic neuropathy

- Neuropathies associated with paraproteinemia. Ropper AH, Gorson KC. N Engl J Med 1998;338:1601-7.

- Neuropathy and monoclonal gammopathy. Nobile-Orazio E, Carpo M. Curr Opin Neurol 2001;14:615-20.

Intravenous immunoglobulin

- Association of British Neurologists guidelines for the use of intravenous immunoglobulin in neurological diseases (March 2002).

Useful information on indications, adverse effects, monitoring, consent and differences between brands. Text may be downloaded from www.theabn.org

- Mechanism of action of intravenous immunoglobulin and therapeutic considerations in the treatment of autoimmune neurologic diseases. Dalakas MC. Neurology 1998;51(6 suppl 5):S2-8.

A review of probable mechanisms of action of intravenous immunoglobulin.
Focal peripheral neuropathies

- Mononeuropathies: examination, diagnosis and treatment. Staal A, van Gijn J, Spaans F. London: WB Saunders, 1999.

- Focal peripheral neuropathies, 3rd ed. Stewart JD. Lippincott, Williams, Wilkins, 2000.

Two excellent summaries of all focal neuropathies. Know where to find a copy of one of these references.

\section{Muscle disease}

- Disorders of voluntary muscle, 7th ed. Karpati G, Hilton-Jones D, Griggs RC, eds. Cambridge University Press, 2001.

A comprehensive source of information about muscle diseases.

\section{Erratum}

Schott JM, Fox NC, Rossor MN. Genetics of the dementias. J Neurol Neurosurg Psychiatry 2002;73(suppl II):ii27-31. In this article, it was stated that the error size of the prion protein gene was $100 \mathrm{bp}$ when it is approximately $750 \mathrm{bp}$.

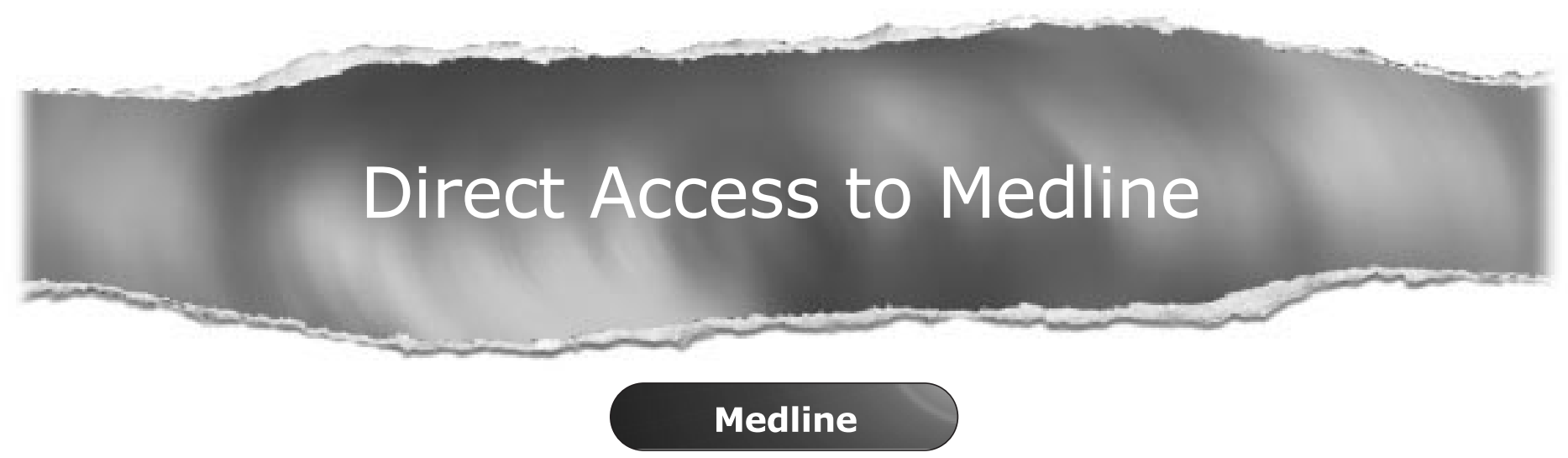

Link to Medline from the homepage and get straight into the National Library of Medicine's premier bibliographic database. Medline allows you to search across 9 million records of bibliographic citations and author abstracts from approximately 3,900 current biomedical journals.

\section{www.jnnp.com}

